stellt eine nicht unerhebliche Investition dar. Wenn die WLB - wie es in einem Strategiepapier des Landes vorgesehen ist - zum Kompetenzzentrum ausgebaut wird und als Dienstleister für kleinere Einrichtungen ohne eigene Infrastruktur auf den Plan tritt, wird die Arbeit mit dem Scanroboter von entscheidender Bedeutung sein.

Christian Herrmann

Dorothea Reinhold

\title{
Die Plakatdatenbank der Bibliothek für Zeitgeschichte
}

\section{Die Plakatsammlung der Bibliothek für Zeitgeschichte}

In den Sondersammlungen der Bibliothek für

Zeitgeschichte (BfZ) finden sich auch zahlreiche

Plakate. Die ursprüngliche „Kriegssammlung” aus dem Ersten Weltkrieg, die 1923 ca. 15.000 Bildplakate verschiedener Länder umfasste, dürfte eine der größten Sammlungen der damaligen Zeit gewesen sein. ${ }^{1}$ Zeitgenössische Berichte über die Sammlung und Fotos der Ausstellungsräume des 1933 eingerichteten Museums mit Beständen der „Weltkriegsbücherei“" geben einen guten Eindruck von der Reichhaltigkeit der Plakatbestände zu damaliger Zeit. ${ }^{2}$
Eine Erweiterung erfuhr die Sammlung durch die Eingliederung von etwa 10.000 Plakaten aus der Kriegssammlung der Hofbibliothek Stuttgart im Jahr 1938. ${ }^{3}$

Die so vergrößerte Plakatsammlung der Weltkriegsbücherei umfasste 1944 ca. 30.000 Stück; sie wurde im selben Jahr bei einem Luftangriff auf das Schloss Rosenstein vollkommen zerstört. Nach dem Zweiten Weltkrieg wurde damit begonnen, die Plakatsammlung durch antiquarische Käufe neu aufzubauen. So erwarb man beispielsweise schon 1952 ca. 400 Plakate aus der Sammlung von Theodor Lach in Graz. ${ }^{4}$ Heute umfasst die Sammlung der BfZ ca. 30.000 Plakate, davon

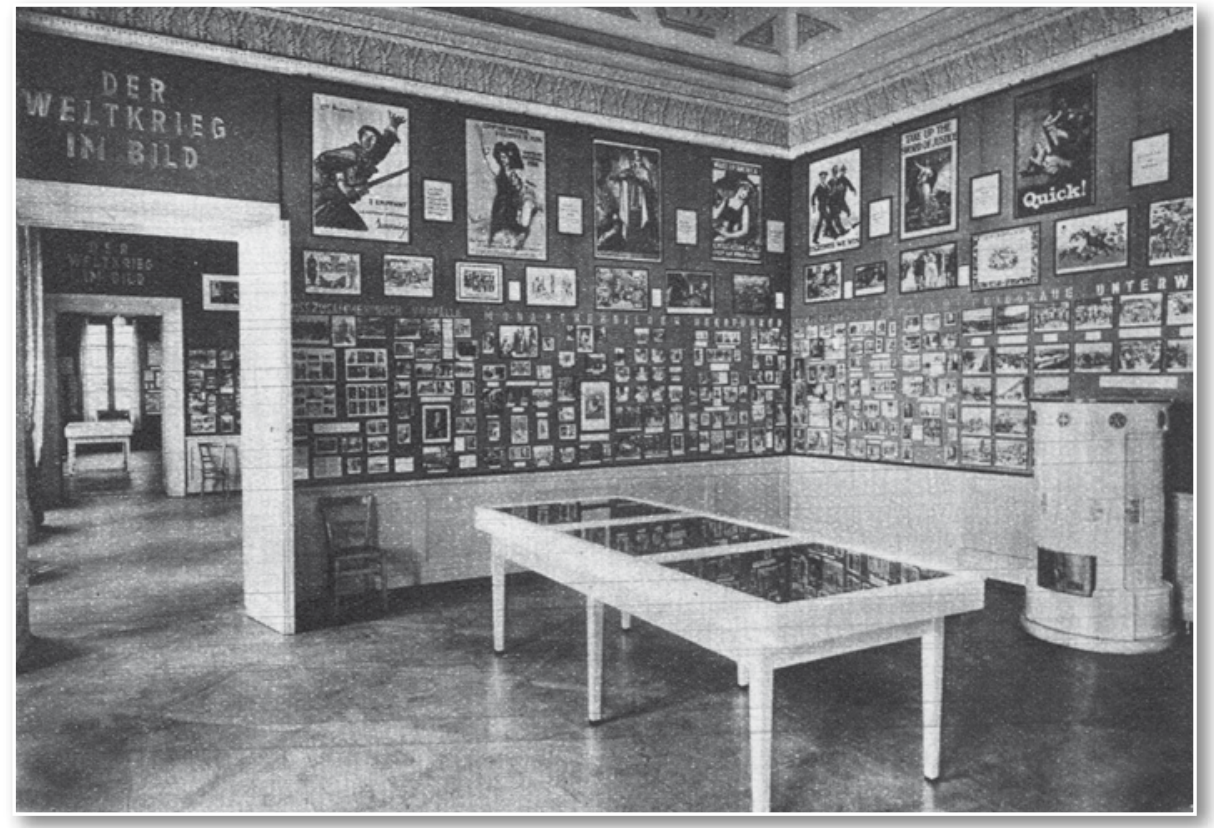

ca. 4.300 aus der Zeit des Ersten Weltkriegs, der Weimarer Republik und des Dritten Reiches. Unter ihnen befinden sich auch zahlreiche Bekanntmachungen aus den Gebieten, die während des Ersten Weltkriegs von Deutschen besetzt waren. Der Großteil der Plakate - ca. 25.000 - stammt jedoch aus den Beständen der ehemaligen „Dokumentationsstelle für unkonventionelle Literatur", der heutigen Sammlung "Neue Soziale Bewegungen“.
Ausstellungsraum zum Thema "Der Weltkrieg im Bild" in der alten "Weltkriegsbücherei" mit zahlreichen ausgestellten Plakaten, aus: Funk-Illustrierte für Süddeutschland, Nr. 26, 23.6.1934
(1) Berichte der Weltkriegsbücherei, Heft 2, Februar 1924, S. $7 f f$.

(2) Siehe z. B. eine Abbildung in: Funk-Illustrierte für Süddeutschland, Nr. 26, 23.6.1934.

(3) Akten der BfZ, Archiv, IV-4 „Berichte über fremde Institutionen und Persönlichkeiten."

(4) Akten der BfZ, Archiv der BfZ.

Zum Werbearchiv von Theodor Lach siehe: Sülzen, Burkhard: Das Werbearchiv Direktor Theodor Lach in Graz, in: Doosry, Yasmin u.a. (Hg.): Plakativ! Produktwerbung im Plakat 1885-1965; die Nürnberger Plakatsammlung - eine Stiftung der GfK und der NAA im Germanischen Nationalmuseum, Ostfildern: Hatje Cantz 2009, S. 38-55. 
Hierbei handelt es sich um Plakate zu den Themen Studentenbewegung, Anti-Atomkraft-Bewegung, Umweltbewegung u.a. Die „Dokumentationsstelle" sammelte seit 1972 auf Anregung und mit Unterstützung der DFG derartige „unkonventionelle“ Materialien, darunter auch zahlreiche Plakate aus dem Zeitraum 1965 bis 2005.

\section{Bismas-Datenbank}

Die Plakat-Bestände der Sammlung "Zeitalter der Weltkriege" wurden schon seit Anfang der 1990er Jahre systematisch in einer Bismas-Datenbank erschlossen, ebenso kleine Teile der Plakate der Sammlung „Neue Soziale Bewegungen“. Die Datenbank enthielt über 4.500 Datensätze mit z. T. sehr detaillierten Angaben zu den einzelnen Plakaten.

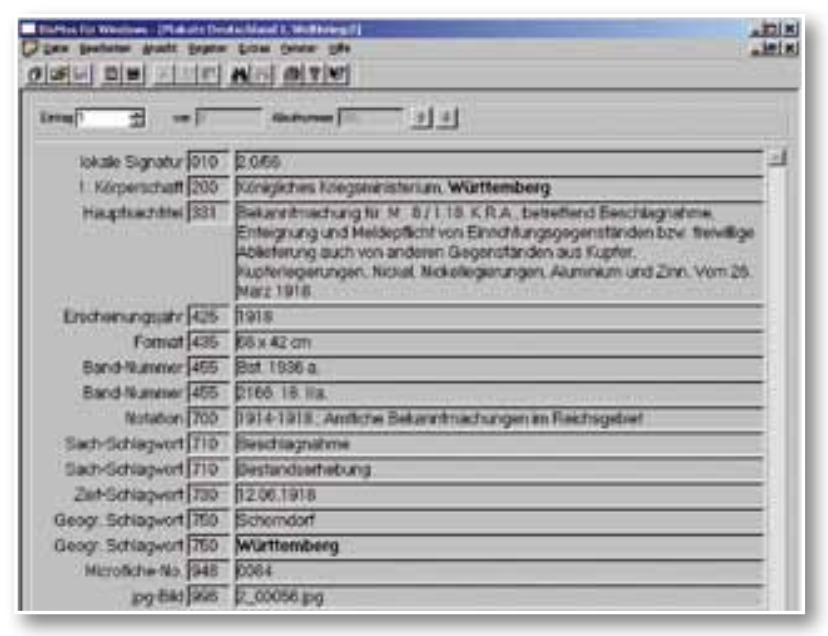

Volltitel-Anzeige in der Bismas-Version der Plakat-Datenbank

Das Datenbanksystem Bismas ${ }^{5}$ wurde Ende der 1980er Jahre von der Universität Oldenburg entwickelt und in einer ersten Version 1991 ausgeliefert. Das Programm entstand zunächst für das Betriebssystem MS-DOS; rund 10 Jahre später erschien eine Weiterentwicklung für die WindowsBetriebssysteme.

Das Datenbanksystem Bismas eignet sich zur Erfassung von beliebigen, kategorisierten Daten, wie sie insbesondere in Bibliotheken verwendet werden. Es unterstützt die Verwendung gängiger Bibliotheksformate, kann aber auch mit eigenen Kategorienformaten verwendet und somit an die konkreten Bedürfnisse einer Einrichtung angepasst werden. Damit eignet es sich für praktisch jede strukturierte Sammlung von Daten.
Die Weiterentwicklung des Datenbanksystems Bismas wurde vor einigen Jahren eingestellt. Um die Plakat-Datenbank künftig sicher betreiben zu können - nicht zuletzt bei einer geplanten Umstellung auf ein aktuelles Windows-Betriebssystem -, wurde die Entscheidung getroffen, sämtliche Daten in eine Allegro-Datenbank zu migrieren. Damit bietet sich nun auch die Möglichkeit, die Datenbank online zu präsentieren; bis jetzt war online nur ein Abzug der BfZ-Plakatdatenbank im "Themenportal Erster Weltkrieg" bei clio-online recherchierbar, hier jedoch ohne Abbildungen. ${ }^{6}$ Schon die Bismas-Datenbank bot jedoch Verknüpfungen mit Abbildungen von Plakaten. Da zahlreiche Plakate der Sammlung "Zeitalter der Weltkriege" in den letzten Jahren im Rahmen des Landesrestaurierungsprogramms restauriert und sicherheitsverfilmt werden konnten, liegen viele ältere Plakate auch als Farb-Macrofiches vor. Von diesen Macrofiches aus wurden dann Gebrauchsdigitalisate in geringer Auflösung angefertigt und mit den Einträgen in der Bismas-Datenbank verknüpft. Da man im Rahmen des Landesrestaurierungsprogramms in den letzten Jahren auch dazu überging, hochwertige Digitalisate anzufertigen, existieren von einigen dieser älteren Plakate auch entsprechende hochwertige Digitalisate.

\section{Datenbank allegro-C}

Das Datenbanksystem allegro-C ${ }^{7}$ wurde ab 1980 an der Universitätsbibliothek Braunschweig entwickelt. Es bietet wie Bismas die Möglichkeit, neben vorgegebenen Kategorienformaten eigene Schemata zu definieren. Damit besteht auch hier die Möglichkeit, das Datenbanksystem für nahezu beliebige Zwecke zu verwenden.

Das System allegro-C wird laufend weiterentwickelt. Neben Programmen für aktuelle Windows-Systeme gibt es aktuelle Varianten für die Betriebssysteme Linux und Solaris sowie den Einsatz als Web-Anwendung. Mit der zugehörigen Skriptsprache FLEX besteht die Möglichkeit, eigene Aufgaben zu automatisieren. Die sehr mächtige (Export-)Parametersprache ermöglicht Ausgaben für die unterschiedlichsten Zwecke: von der Bildschirmdarstellung im Windows-Client oder im Browser über den Zetteldruck bis zum Export einzelner oder aller Daten in den unterschiedlichsten Varianten.

(5) Bibliographisches Informationssystem zur maschinellen Ausgabe und Suche.

(6) http://www.erster-weltkrieg.clio-online.de/default. aspx?tabid $=40208181$

(7) http://www.allegro-c.de 
In der Württembergischen Landesbibliothek wurde bereits in der Bismas-Version der Plakat-Datenbank ein dreistelliges Kategorienformat verwendet, das an das MAB-Format angelehnt war, jedoch an die Bedürfnisse der Plakaterschließung angepasst wurde. So sind die grundlegenden Kategorien für Titel und Autoren übernommen worden. Diese werden in der Darstellung im Webbrowser mittels der Exportparameter jedoch in ihrer auf die PlakatDatenbank abgestimmten Bedeutung angezeigt: Statt "Autor" erscheint „Entwurf", statt "Verlag" "Verlag/Druck".

Die Erfassung neuer Datensätze erfolgt in der WLB ausschließlich über ein Webformular. Dadurch ist es ohne Zusatzprogramme möglich, Unicode-Zeichen einzugeben und direkt in der richtigen Darstellung anzuzeigen, denn die Datenbank verwendet in der hier genutzten Version im Windows-Client eine interne Darstellung in Form von HTML-Entities für nicht-deutsche Schriftzeichen. Die Eingabemöglichkeiten der Webprogramme wurden in der WLB erweitert, sodass über eine Javascript-Anbindung häufig vorkommende Textbausteine in den Datensatz übernommen werden können. Außerdem wurde eine Funktion zur Hervorhebung der gesuchten Begriffe im Suchergebnis ( „,highlighting“) implementiert. Dadurch wird sofort sichtbar, in welchen Kategorien eines Treffers die gesuchten Begriffe gefunden wurden.

Bei der neu entstandenen Datenbank kann man eine externe und eine interne Version unterscheiden. Nur in der internen Version ist die Eingabe und Korrektur von Datensätzen möglich. Hier gibt es auch umfangreichere Such- und Exportmöglichkeiten. Außerdem werden in dieser internen Version alle vorhanden Abbildungen angezeigt. In der externen, also der nach außen sichtbaren Version der Datenbank, gelangt man unter „Neue Suche“ zur Suchmaske der Plakatdatenbank, die neben der Volltextsuche auch gezieltere Suchanfragen nach Titel, Entwerfer, Auftraggeber/Herausgeber, Erscheinungsjahr, Verlag/Druck, Schlagworte, Exakter Titel, Entwerfer (Name, Vorname) und Signatur erlaubt. Außerdem sind der Registereinblick sowie die Kombination von Suchbegriffen möglich.

Die Allegro-Datenbank vereint die ehemals 21 getrennt angelegten Plakat-Datenbanken zu verschiedenen Gebieten. In der Suchmaske lässt sich in der Zeile "Land einschränken“ die Suche weiter fokussieren, beispielsweise auf „Deutschland Erster Weltkrieg", "Deutschland nach 1945", „Österreich“ oder auch „Großbritannien“. Da einige dieser Bereiche recht umfangreich sind, gibt es in einigen Gruppen die Möglichkeit, weitere Teilbereiche einzuschränken, so zum Beispiel im Bereich „Österreich" die Gruppe „1914-1918; Kriegsanleihe, Sammlungen“ oder "1919-1937; Veranstaltungen". Man kann sich nun sämtliche Plakate aus diesen Gruppen anzeigen lassen oder auch innerhalb der einzelnen Gruppen recherchieren. Die Informationen zu den vorhandenen Gruppen werden täglich automatisiert ausgewertet und für die Bildschirmdarstellung aufbereitet.

Schickt man die Suchanfrage ab, gelangt man zu einer Kurztitelliste. Im oberen Bereich wird die Anzahl der Treffer angezeigt, außerdem hat man die Möglichkeit, mit „Gehe zu“ zu einem bestimmten Treffer zu springen. Die Kurztitelliste präsentiert die ersten 40 Treffer einer Suchanfrage. Angezeigt werden die Abbildung (soweit vorhanden und urheberrechtlich frei) sowie die Kategorien Titel, Entwurf, Datierung, Signatur und Macrofiche-Nummer (wenn vorhanden). Die Suchbegriffe werden sowohl in der Kurz- als auch in der Vollanzeige "gehighlightet".

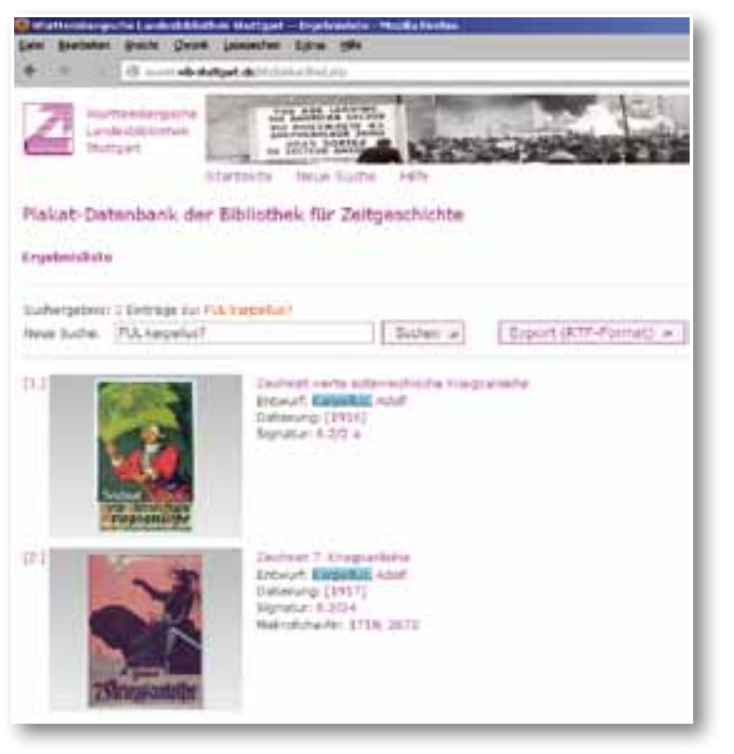

Kurztitelliste der allegro-C-Version der Plakatdatenbank der BfZ

Da die abgebildeten Plakate unterschiedliche Formate haben und diese keinesfalls verzerrt aber dennoch gleich groß angezeigt werden sollten, wurde die Anzeige so gestaltet, dass jedes Plakat in ein vordefiniertes, immer gleich großes Fenster eingepasst wurde, womit gleichzeitig ein ruhiger 
optischer Eindruck in der Anzeige erzielt werden konnte. Um besonders lange Listen, in denen keine oder nur wenige Abbildungen vorhanden sind, etwas abzukürzen, wurde die Größe dieser Felder verringert, wenn keine Abbildungen vorhanden sind oder diese nicht angezeigt werden dürfen. Sowohl die Abbildungen als auch die Kurztitel-Beschreibungen sind verlinkt mit dem Haupteintrag. Hier werden alle relevanten Daten zum jeweiligen Plakat sowie eine größere Abbildung (wenn vorhanden) angezeigt. Die Abbildungen lassen sich in einem weiteren Schritt als jpg-Dateien in größtmöglicher Auflösung anzeigen.

Um Ergebnislisten abspeichern oder ausdrucken zu können, enthält die Datenbank in der Kurztitelliste eine Exportfunktion. Hier wird der automatische Export der Daten in eine rtf-Textdatei angestoßen, die neben der Treffernummer die Kategorien Titel, Entwurf, Datierung, Signatur und Macrofiche-Nummer enthält. Diese Funktion musste für das eigene Kategorienformat neu entwickelt werden.

\section{Rechtliche Aspekte}

Die Datenbank enthält aktuell Datensätze zu 4.607 Plakaten (Stand: 7.1.2013), bei 2.591 ist eine Abbildung vorhanden. Außer den Schriftplakaten, die als amtliche Veröffentlichungen gemeinfrei sind, sind viele Bildplakate als eigene schöpferische Leistung des Entwerfers urheberrechtlich geschützt. Diese Frist endet erst 70 Jahre nach dem Tod des Urhebers, so dass für zahlreiche Plakate auch heute noch ein Urheberrechtsschutz besteht. Zu jedem Datensatz wurde dementsprechend ein neues Feld "Bildrechte" hinzugefügt, in das entweder das Todesjahr des Künstlers, der Vermerk "Schriftplakat" oder der Vermerk „unbekannt" eingetragen wurden. Der Vermerk „unbekannt" wurde gewählt, wenn entweder das Todesdatum des Künstlers oder auch der Künstler selbst nicht zu ermitteln waren. Für Plakate, die nach 1945 erschienen sind, wurde bis jetzt keine Eintragung vorgenommen, es sei denn, das Todesdatum des Künstlers war eindeutig bekannt. Die Plakate mit den Vermerken "Schriftplakat" oder „unbekannt" sowie die Plakate, deren Urheber vor mehr als 70 Jahre gestorben sind, werden im Internet frei angezeigt. Bei allen anderen erscheint der Hinweis "Aus urheberrechtlichen Gründen darf dieses Bild nicht angezeigt werden", auch bei denjenigen Plakaten, die überhaupt keine Angabe im Vermerk „Bildrechte“ enthalten. Unter diesen Voraussetzungen können wir derzeit 2.202 der 2.591 Abbildungen anzeigen lassen. Durch die Eintragung des Todesdatums des Entwerfers werden mit Beginn jedes neuen Jahres automatisch weitere Abbildungen zur Anzeige frei gegeben. Außerdem ließen sich so bei einer eventuellen Änderung der Schutzfristen die AnzeigeModalitäten leicht anpassen.

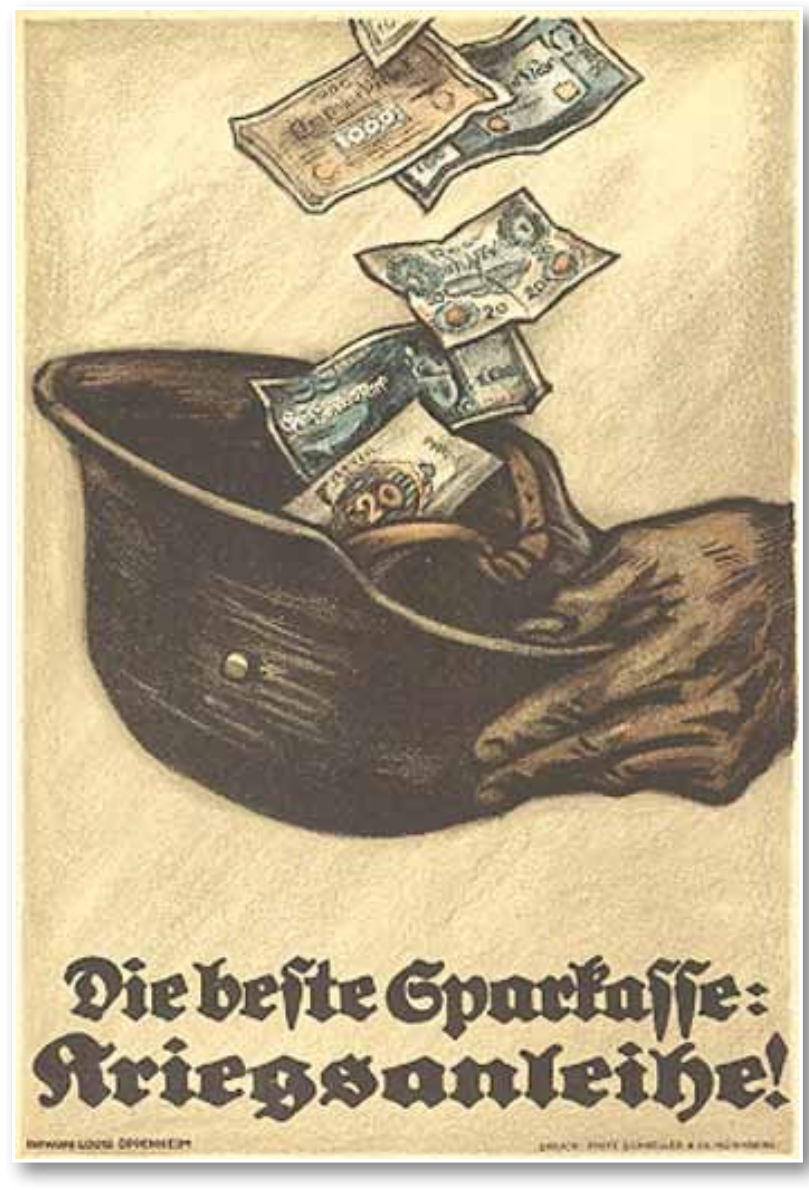

Louis Oppenheim (1879-1936): „Die beste Sparkasse: Kriegsanleihe”, Plakat zur achten deutschen Kriegsanleihe 1918, eines der gemeinfreien Künstlerplakate

Das Feld „Bildrechte" wird in jeder Nacht exportiert und automatisch ausgewertet. Falls ein positives Bildrecht angegeben ist, wird anschließend das Bild auf den Webserver kopiert sowie ein Vorschaubild (Thumbnail) erzeugt. Ist das Plakat nicht urheberrechtsfrei, werden dagegen lediglich Ersatzbilder mit Urheberrechtsvermerk anstelle der Originalbilder auf den Webserver gestellt.

Der Zugang zum urheberrechtlich geschützten Material kann nach wie vor nur in den Räumen der Sammlungen der Bibliothek für Zeitgeschichte erfolgen, wo die Originale der Plakate oder gegebenenfalls auch Farbmacrofiches einzelner Plakate eingesehen werden können. 


\section{Ausblick}

Die Plakat-Datenbank kann in der nun vorliegenden Form problemlos erweitert und ergänzt werden. Die noch nicht erfassten Plakate - insbesondere aus der Sammlung "Neue Soziale Bewegungen" sollten in der Datenbank neu erfasst werden. Möglichst alle Plakate sollten dann mit einem Digitalisat ergänzt werden. Zu überlegen wäre auch, ob man den Ausschnitt zum Themenbereich Erster Weltkrieg wieder - wie bei der alten Version - über das "Themenportal Erster Weltkrieg" von clio-online anbietet. ${ }^{8}$
In den Sammlungen der Bibliothek für Zeitgeschichte wurde das Datenbanksystem Bismas auch zur Erschließung anderer Materialien eingesetzt, so sind beispielsweise Bismas-Datenbanken zu Fotos, Flugblättern oder Lebensmittelkarten vorhanden. Auch diese sollten nach dem Muster der Plakat-Datenbank nach allegro-C migriert und über das Web angeboten werden.

Zu überlegen wäre die Vereinigung aller dieser Datenbanken in einer großen Bild-Datenbank, möglichst für alle Sondermaterialien der Württembergischen Landesbibliothek.

Jörg Oberfell

Hans-Christian Pust

\section{Informationskompetenz für Schüle- rinnen und Schüler der Oberstufe}

In den Wintermonaten wird die WLB traditionell sehr stark von Schulklassen besucht. Ein Blick auf den Belegungsplan des Schulungskalenders zeigt, dass auch die Vermittlung von Informationskompetenz in den letzten Monaten des Jahres ganz im Zeichen der Oberstufenschüler stand: Mit der Schulung "Fit für Seminarkurse”, dem Rechercheworkshop „Fit ins Abitur" sowie der Lehrerschulung bot die WLB im Jahr 2012 das zweite Jahr in Folge ein stark zielgruppenorientiertes Schulungskonzept für Schülerinnen und Schüler der gymnasialen sowie beruflichen Oberstufe an, welches ausgesprochen gut nachgefragt wurde.

\section{Zielgruppen und Nachfrage}

Die ab 2004 sukzessive in Kraft getretenen neuen Bildungspläne mit ihrer starken Akzentuierung von Kompetenzen (insbesondere fachliche, personale, soziale und methodische Kompetenzen) messen dem selbstgesteuerten Lernen eine wichtige Bedeutung zu. Spätestens bei der Vorbereitung der Präsentationsprüfung (fünftes Prüfungsfach im mündlichen Abitur), vielfach aber schon ein Jahr früher im Seminarkurs, welcher alternativ dazu erbracht werden kann, werden die Schülerinnen und Schüler mit Arbeitsmethoden konfrontiert, die in dieser Form früher erst im Studium oder Berufsleben gefordert wurden: Für die Präsentationsprüfung reicht der Schüler vier Themenvorschläge ein, aus denen der Prüfungsvorsitzende eines auswählt. Nach Bekanntgabe des Themas hat der Prüfling ca. eine Woche Zeit, um dieses inhaltlich zu bearbeiten und eine Präsentation dazu zu erstellen. Die mündliche Abiturprüfung im fünften Prüfungsfach besteht aus dem etwa zehnminütigen Referat des Prüflings sowie Fragen zum Referat, dem verwandten Sachgebiet und anderen im Unterricht behandelten Themen durch die Mitglieder der Prüfungskommission.

Eigenverantwortliches Lernen - im Gegensatz zum rein rezeptiv-reaktiven Aufnehmen von Lernstoffen - spielt auch im Seminarkurs eine große Rolle. Ähnlich wie in der Präsentationsprüfung ist selbständiges Recherchieren von Informationen unabdingbar: So muss im Seminarkurs eine schriftliche Dokumentation, möglichst auf der Basis von Literaturrecherchen in einer wissenschaftlichen Bibliothek, angefertigt und in einem Kolloquium vorgestellt werden. Seminarkurse sind stets interdisziplinär ausgerichtet und entweder dem sprachlich-literarisch-künstlerischen, 